\title{
Digital Society Artificial Intelligence in Health Care: Issues of Legal Ethical and Economical Sustainability
}

\author{
Dr. Kyvalya Garikapati ${ }^{1}$, Khushi Shaw ${ }^{2}$, Ankita Shaw ${ }^{3}$, Dr. Anuradha \\ Yarlagadda $^{4}$
}

${ }^{1}$ KIIT University, kgarikapati@kls.ac.in

${ }^{2}$ Student, The Heritage College, shaw.khushis@gmail.com

${ }^{3}$ Student, KIIT University,1683023@kls.ac.in

${ }^{4}$ Gayatri Vidya Parishad College of Engineering (A), anuradhayarlagaddag@gmail.com

"Corresponding author.Email: kgarikapati@kls.ac.in

\begin{abstract}
Artificial Intelligence (AI) being the most transformative technology of $21^{\text {st }}$ Century has paved new frontiers in every field. One of the early candidates to be revolutionized was Health Care. The implementation of AI has an immense promise to improve the health outcomes in a resource-constrained setting like India although with limitations. The data by Central Bureau of Health Intelligence estimated the total size of health care workers in India as 4.7 million in 2015. The objective behind implementing AI was to ease down the pressure of the health care staff, lower down costs, and improve the lives of the patients. It is welcoming to implement AI in Health Care for it influences global sustainability, however, these implementations of AI in Health Care have ramifications in legal, ethical and economic sustainability. With AI transforming the world, the pertinent issue that can be questioned is, it may ensure a utopian future where the harmonization of the co-existence of human and machines would take place or such developments would portend a world with all kinds of divide the consequence of which would be poverty and suffering. The issues about the development of AI on the imminent note are, whether the development of AI in health care leads to economic sustainability? Furthermore, with the backdrop of the framework existing in India, would such development in health care lead to economic, legal and ethical ramifications? Lastly, whether such developments would bring progress towards United Nations sustainable development goals or pave us towards greater economic havoc and collapse of the environment? The article aims to address these issues by analyzing the impacts of AI in multifarious sectors. The study combines the legal and ethical ramifications of implementing Artificial Intelligence in Health Care with an advancement of the SDGs and its impact on economic sustainability.
\end{abstract}

Keywords: Artificial Intelligence, Economic, Health, Legal, Sustainability.

\section{INTRODUCTION}

The previous concept of Artificial Intelligence (AI in brief) was consigned to futuristic societies which were but engendered in the fictional stories in the movies and motion pictures. This has transformed into reality in the 21 st Century in the form of Artificial Intelligence. AI has gained momentum in day to day life of every individual sanctioning it to become one of the most technologically advanced societies when compared to the previous kinds of an era that subsisted.
Previously, considered as one of the utopian concepts, AI today, in reality, has increased ubiquitously in every arena of the human world which is transforming every conventional aspect. AI has the potential to revolutionize the way we discover, learn, live, communicate, and work? (A. Goralski and Tan, 2020). In simplified terms, AI means that it resolves complex cognitive problems that are associated with human intelligence. It is also considered that AI helps as many people as possible through smartphones or health care systems. The crux of AI is considered to be such an aspect that creates machines that has the capability of cognitive 
thinking just like human beings or even more than that.

With AI starting to become the torch-bearer in every sphere, the Government officials, policymakers, health care staff, and others are becoming highly concerned that with this level of advancement, it won't be long when human beings will be replaced and superseded by such AI Machines. Stephen Hawking, a physicist, and a cosmologist once opined that humans with their slow biological system will not be able to compete with intelligent machines and therefore, could be easily replaced by them.

As the world expeditiously unfolds to welcome AI in every possible domain, the paper focuses on the issue of ethical, legal, and economic sustainability due to the implementation of such machines in healthcare.

\section{THE POTENTIALITY OF AI IN HEALTHCARE IN INDIA}

The doctor-patient ratio in India is $1: 10,189$ (Frost, Isabel, and Craig, 2019) which is considered to be 10 times shorter than what has been suggested by the World Health Organization. Furthermore, with the constraint in resources, there has never been a louder call for implementing $\mathrm{AI}$ in healthcare.

The Healthcare landscape is considered to be one of the steadiest fields which have embraced the digital health innovation to respond to the critical challenges of health care. India in the past decennium has witnessed an increase in the utilization of AI based machines in healthcare. For example, the Siemens Healthineers has taken a step to offer a range of AI products to standardize and automate complex diagnostics and meet patient needs. Furthermore, even Microsoft has tied up with Apollo to provide an AI-centric cardiology network. Albeit, the start-up companies have taken the initiative to develop and implement AI in healthcare, the louder call is to not overlook ethical, economic, and legal repercussions that it causes.

\section{ETHICAL IMPLICATION OF THE USE OF AI IN HEALTHCARE}

The use of AI in healthcare and the medical landscape is a developing one however, even at a nascent stage of its application, it has proved to be consequential in various perspectives. One of the pertinent and significant issues is that of ethical implication. It is to be accentuated that healthcare decisions in the past have always been made by human beings thus, with the use of AI in healthcare, it imminently raises the issues of accountability, transparency, data protection, privacy, informed consent, and permission.

\subsection{Transparency}

Perhaps, to understand the ethical perspective, it becomes imperative to understand that one of the most difficult issues is that of transparency. Given that the algorithms used by AI in healthcare are that of image analysis, it becomes virtually infeasible to interpret or explain the same. Even the physicians who are familiar with the operational framework of the same may find it difficult to interpret and analyze the same to the patients.

\subsection{Accountability}

Another paramount ethical issue is that; it needs to be understood that the AI systems will undoubtedly make mistakes in patient diagnosis. Furthermore, this mistake may lead to difficulty in establishing accountability. Furthermore, there may also be instances wherein when patients receive medical information from AI Systems about their medical quandaries which they would like to verify or would prefer to receive the same diagnosis from an empathetic medical health care staff.

\subsection{Algorithmic Bias}

It is additionally to be understood that the algorithms which are used by AI may also be biased, which in all likelihood would consequently predict the disease based on gender or race when those are not considered to be causal factors. (TH and K.A., 2018) There have been various examples wherein it has been demonstrated that algorithms can exhibit biases that can result in injustice in the context of ethnicity, origin, and skin color or gender. (Cossins, 2018) In the landscape of healthcare wherein, certain genotype diseases do exist, biased AI could and in all possibility be a leading factor towards mendacious diagnosis and render treatments ineffective for some of the sub-population and thus lead to jeopardizing their safety.

\subsection{Informed Consent}

This has become one of the pressing issues to understand the ethical challenges that are posed by AI in healthcare, however, has been the least considered aspect in the review. The issue pertinent 
on this note is what if at all, is the liability and obligation of the clinicians and health care staff to expound and elaborate to the patients the complexities and intricacies involved in the use of AI, including the forms of Machine Learning used by the system? Furthermore, under what circumstances must a health care staff inform the patient that $\mathrm{AI}$ is being used at all?

Be In a developing nation like India, with the start of the use of apps like Aarogya Setu and several others health apps and chatbots ranging from health guidance to assessments to the help to improve medication, the issue of informed consent becomes all the more pertinent considering the user agreements and their relationship to informed consent. In a nation like India where the literacy level is merely $74.04 \%$ and more than half the population depending on rural life, expecting these individuals to take time and understand the complexities of such agreements (Klugman C.M., 2018, Suar \& Samanta, 2020) or for that matter of fact even understand the agreement is like expecting an individual who is not a doctor to know the causes for a disease. Moreover, frequent updates of the software make it all the more difficult for an individual to understand the terms of service they followed (M. T. Gerke S., 2019). The pertinent issue is how many individuals per se sufficiently and substantially understand the future use of such apps and chatbots may be conditional on accepting changes to the terms of use. Furthermore, the question also is what would an ethically responsible user agreement looks like in this context (Sara Gerke, 2020).

\subsection{Safety of the use of AI}

One of the biggest challenges of the use of AI in health care is that of safety. One such example is that of IBM Watson for Oncology. In the instant case, it uses algorithms to access the information from patients' medical records and as well helps physicians explore cancer treatment options for the patients. However, recently, it has been criticized for reportedly giving "unsafe and incorrect" recommendations for cancer treatments (J. Brown, 2018). The considerable problem was with the training of the software with a few "synthetic" cancer cases.

\section{ECONOMIC CONSTRAINTS IN THE IMPLEMENTATION OF AI IN HEALTHCARE}

One of the pertinent issue regarding the implementation of AI in the healthcare landscape in India is the issue of economic sustainability. There are various aspects of the Indian socio-economic framework that not only implements AI difficult but also questions if the implementation will be bearable and in consonance with the United Nations sustainable development goals.

\subsection{The issue of Economic Growth vis-à-vis Economic Sustainability}

"Economic sustainability focuses on meeting the needs of the present without compromising the ability of future generations to meet their needs." In simplified terms, the conceptual understanding of sustainability is to provide quality and adequate life to the present generation while ensuring a decent living for future generations.

When we alter the definition of economic sustainability it envisages that to bring about economic sustainability it becomes imperative for a nation to have a $2 \%$ steady growth. However, this definition per se cannot be considered because it fails to provide information about the individuals at the extreme ends of the income distribution.

Due to the lack of a proper alternate definition of economic sustainability, the concept of poverty threshold is, therefore, put forth to bring clarity to economic sustainability. India's poverty threshold is Rs. 32 per day and Rs. 26 per day for urban and rural areas respectively. The poverty threshold is the minimum income which defines an adequate standard of living, which is considered to be very low. We may now include this in the definition discussed above, and the new definition as altered may be verbalized in the following words "Economic sustainability refers to growth inclusive of practices that support the preferred percentage of its population below its preferred minimum standards of living level, without negatively impacting social, environmental and cultural aspects of the community." (Courtnell, 2019) In simplified terms, the preferred percentage of people living below the given poverty line should be minimum which will in turn ensure economic sustainability.

India is a developing country and to achieve economic sustainability, the standard of living will have to increase, human beings will have to 
habituate to new ways of life, for which economic growth and development for all is necessary. To bring sustainability, economic growth is indispensable however, vice-versa does not hold which has been depicted in the given diagram that economic growth leads to economic sustainability.

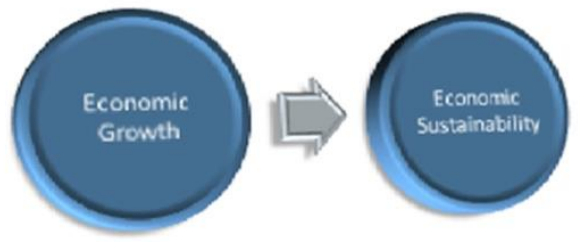

To analyze the perspective of establishing economic sustainability in the healthcare sector it is to be emphasized that the government of India approximately spends $1.28 \%$ of the total GDP in the healthcare sector which when compared to countries like the USA $(17.7 \%)$, China $(6.57 \%)$, Singapore $(4.4 \%)$ is very low. The most important work to be done is to increase the percentage of GDP spent in the healthcare sector to establish the implementation of AI. India being an agro-based country and not so established in research and development will depend mostly on other countries for the advancement and inclusion of AI in the health care sector, which will only be possible if the amount allocated to the healthcare sector increases. However, for these facilities to be relished by everyone all over the country, there are other issues which will have to be eradicated like firstly, the number of individuals below the poverty line must be decreased to a minimum level, secondly, people will have to get used to digitalization and thirdly, literacy level should be incremented for ample understanding of the AI-based technology only after which it can become economically viable. Otherwise, the problems of accessibility, affordability, equitability will crop up resulting in the consumption of these benefits by a limited number of individuals.

\subsection{Economic Repercussions with the introduction of AI in health care}

India is a developing nation that is subject to problems like unemployment, poverty, illiteracy in all the sectors. The health care system is also not immune to these issues. With the introduction of AI, the decision-making techniques become more accurate and precise, it also truncates the encumbrances on the working healthcare staffs, and also reducing the risk to a certain extent relating to diseases. However, in a country like India, the economic viability and the equitability of the same is questionable.

\subsubsection{Issue of achieving the set Sustainable Development Goals due to economic repercussions}

Delving into the problem of equitability it is to be understood that $\mathrm{AI}$ in the health care sector to some extent will bring about good health and well-being with its precision and medical insights which will be in line with the SDG 3, however only $11 \%$ subcenters, $13 \%$ Primary Health Centres (PHCs) and $16 \%$ Community Health Centres (CHCs) in rural India meet the Indian Public Health Standards (IPHS), so this facility will not be available to most of the population of India in the rural areas, thus not achieving the Sustainable Development Goal 10 of reducing inequality. People living in suburban areas and rural areas have to travel to major cities providing the AI-based healthcare facilities to be able to gain access to the same and as a result, spending more than they can afford on the same. Also, considering that the poor are more likely to be sick owing to the lack of proper nutrition, unhygienic surroundings, and several other factors. Hypothetically, if these individuals living below the poverty threshold suffer from cancer and if they are unable to either afford insurance or access the topnotch facilities like AI-based diagnosis, how can it be considered to be economically viable. It will merely increase the chances of the rich getting proper healthcare facilities, and as usual poor lagging behind in one sector which is considered to be one of the fundamental desiderata of every individual.

Another major problem that India would face with the introduction of $\mathrm{AI}$ is the rise of unemployment. The rise of AI will create jobs, but the loss of jobs will be at an unprecedented level, more than what we will be able to comprehend. It seems likely that the automation in the healthcare sector will be those including digital information, radiology, and pathology (Davenport and Kalakota, 2019) as it is still in the nascent stages, however, once there is adequate development of machinery the labor market will witness a significant reduction in the jobs. Healthcare workers may expect a shift in their roles concerning their jobs. According to the American Health Association research, the jobs that are likely to be replaced is that of nonclinical staff by $40 \%$ and clinicians' job by 33\% (Franck, 2017). When various research depicts that there will be onethird (Franck, 2017) or more than one-third loss of jobs (Frey and Osborne) in the United States due to 
automation, the question is with the given socioeconomic condition prevalent in India wherein there subsists lack sufficient skill sets in the majority of the people, to what extent will the loss of jobs take place?

It is to be accentuated that implementation of AI in healthcare is not something which is replacing some jobs by machinery but it is replacing the cognitive capability of human beings with that of intelligent machinery. There is a fundamental difference between eliminating some jobs (which are to be replaced) and eliminating humans in general from the labor market. This replacement does not only involve in removing some jobs, rather it involves the replacement of human beings in general from the labor market leading to rising unemployment which will ultimately result in increasing poverty. This consequence of the implementation of AI in health care does not seem to align with goal 1 that is no poverty and goal 2 that is zero hunger of SDG.

\subsubsection{AI adoption and encumbrances to be borne in the healthcare sector}

In a resource constrained country like India, the implementation of $\mathrm{AI}$ is a big problem as major healthcare facilities provided by various hospitals in India are either small scale or barely qualify to be called large scale when compared to the facilities provided by private healthcare sectors. The transformation of manual work of the healthcare industry to machine-oriented work will only take place if the benefits accrued by the up-gradation of infrastructure is more than the cost of technology adoption. In simplified terms, the technology must be cost-efficient. The repetitive or manual menial work like taking appointments, maintaining records of patients, the work of non-medical staff, clerical works and several others have the highest risk of getting automated, however, it may also be considered that this may at the same time have the lowest risk of AI penetration. India is a labor abundant country where employing labor is easier and cost-efficient than to adopt complex AI-based machinery which may not be worth the cost that has to be borne by healthcare industries. Simultaneously, by employing individuals it may also be ensured, that the employment is stable. Hypothetically, let us consider that the non-medical staff is paid approximately Rs. 15,000 to 20,000. However, if we substitute the non-medical staff with AI-based machinery it might cost a good fortune. Even if, private hospitals can afford to substitute manual work with Automation the problem of distribution of the benefits of the same may not be accrued by every individual concerned.

Consequentially, due to the economic repercussions caused for the implementation of $\mathrm{AI}$ in healthcare, some of the legal rights enshrined in the Constitution of India are also violated as discussed below.

\section{LEGAL RAMIFICATION WITH THE IMPLEMENTATION OF AI IN HEALTHCARE}

The regulatory framework in India is not updated to the extent to include and keep abreast with the developments in AI and Technology. There have been several issues which have arisen since no specific law which envisages the framework to deal with $\mathrm{AI}$ in healthcare precisely.

\subsection{Issue of Liability}

Medical Negligence has been one of the common factors when the arena of Healthcare is intersected with that of Law. The liability of medical negligence is generally implicated in healthcare professionals. There have been several instances of civil and criminal penalties being imposed on medical professionals due to their negligence. However, with the implementation of AI in healthcare, the major threat is on the liability imposed considering that the regulations do not either directly or indirectly distinguish cases based on error or mistake or malfunction or the use of inaccurate data in diagnosis by the technology. Consequently, at present, there exists no liability for the software developer or the program designer. The irony in India is that even if a wrong diagnosis is done by the machines, the liability still lies on the medical professional even if there was a technical glitch or an error in the algorithmic data. This in itself poses a major threat to the ethical challenges of implementing AI in Healthcare.

\subsection{Data Privacy}

There is a lack of adequate data privacy law in India. Although the right to privacy has been recognized as a fundamental right by the judicial precedent of K.S.Puttaswamy v. Union of India (2017) yet the lack of data privacy law in India could result in the data sets exchanged by the patient and the service provider to be leaked to the third party as happened in the case of Matt Dinerstein v. Google 
(2020) where the University shared the inpatient details with Google.

\subsection{The regime of Intellectual Property Law}

In India, the sets of algorithms are not recognized as a patentable product by the Intellectual Property Law. These algorithms form the basis for the functioning of AI. The irony is that even though India is moving towards developing AI, it has specifically envisaged in the Patents Act regarding the exemption of algorithms from being "inventions", therefore, it may not be considered for patent protection. It may, therefore, be said, that these existing frameworks are not in consonance to incentivize the development of AI.

\subsection{Right to Equality}

Article 14 enshrines that the state shall not deny to any person equality before law and equal protection of laws. Furthermore, Article 15 of the Constitution of India stipulates about the prohibition of discrimination on grounds of race, caste, sex or place of birth and Article 16 elaborates on Equality of Opportunity in matters of public employment. These principles may be considered to be the core of Right to Equality concept. However, be it algorithmic biases of the AI or be it the accessibility of the facilities provided by AI in healthcare, both amounts to discrimination. The former being based on gender or race and the latter being concentrated on the economic divide which leads towards the nonaccessibility of the health care facilities.

\section{RECOMMENDATIONS}

The purpose of this paper is to cast light upon the several challenges that India as a developing nation might witness with the adoption of AI-based infrastructure in healthcare, be it in the economic or legal framework that persists in India. Some of the taxing demands that is ought to be fulfilled before the implementation of $\mathrm{AI}$ in healthcare are: that firstly, a strong framework regulating the AI in healthcare must be enacted; that secondly, in order to overcome ethical challenges, the accuracy of data shall be high with the incorporation of labelled data; that thirdly, a gradual adoption of AI based infrastructure is made in the healthcare sector rather than suddenly adopting and implementing $\mathrm{AI}$ in healthcare; that fourthly, for implementing AI in any sector, it is imperative to first take an initiative to increase the gross enrolment ratio in higher education and increase research and development activities the consequence of which shall be transforming of individuals into digital citizens also not leaving them unemployed considering that proper skill based training is imparted to the individuals working in their specific sectors (in the given scenario: healthcare sector); that fifthly, in legal sphere, data protection and privacy are major concerns which ought to be overcome by enacting laws which India is still lacking; that sixthly, legal ramifications must be done away with by ensuring that such implementation is cost effective, accessible to all and such services are provided to all with subsidy ensuring equity and fairness; that seventhly, the budget allocation of funds for the implementation of emerging technologies is required to be increased by the Government of India.

\section{CONCLUSION}

It is important to understand that automation once was considered to be a dream which is gaining momentum in the healthcare sector in recent times, however, the adoption of the same is a big challenge in healthcare. In a developing country like India, where socio-economic-legal-ethical problems persist in every sector, AI might prove to be a solution to these structural problems but, it is to be understood that the process of implementation of the same is highly demanding in nature.

These theoretical concepts look good in pen and paper however, it is difficult to clearly state that what the nation will witness in practicality with reference to implementation of AI based technologies in healthcare. The problems might seem like climbing a mountain but, if we have the correct policies enacted and rights decisions taken at the right time it might not be so difficult to reach the peak and thereby, implement $\mathrm{AI}$ in healthcare to bring greater good to human kind. It is too soon however, to make conclusions or be sure of every interpretation made. With the technological advancement AI may seem to be the correct path to keep up with the fast paced first world countries.

\section{AUTHORS' CONTRIBUTIONS}

The discussions relating to law are done by author 1 and 3, the economics related discussions are done by author 2, the scientific details are covered by author 4 . editing was done by author 3 .

\section{ACKNOWLEDGMENTS}

The Director School of Law Prof. Bhavani Prasad Panda, KIIT DU Bhubaneswar, for 
encouragement and allowing access to Central Library.

\section{REFERENCES}

[1] Angela Spatharou, Solveigh Hieronimus, and Jonathan Jenkins. "Transforming healthcare with AI: The impact on workforce and organizations." (2020).

[2] Artificial Intelligence in Society, OECD (2019).

[3] Fei Jiang, Yong Jiang, Hui Zhi, Yi Dong and Others. "Artificial intelligence in healthcare:past, present and future." Stroke and Vascular Neurology, Vol 2 (2017).

[4] Jatinder Bali, Rohit Garg and Renu $\mathrm{T}$ Bali. "Artificial Intelligence (AI) in healthcare and biomedical research: Why a strong computational/ AI bioethics framework is required." Indian J. Opthalmol, Vol 67, No 1, (2019), 3-6.

[5] Justus Wolff, Josch Pauling, Andreas Keck, Jan Baumbach. "The Economic Impact of Artificial Intelligence in Health Care: Systematic Review." Journal of Medical Internet Research, Vol 22, No 2 (2020).
[6] Margaret A.Goralski and Tay Keong Tan. "Artificial intelligence and sustainable development." The International Journal of Management Education, Vol 18, No 1 (2020).

[7] Petropoulos G. "Do We Understand the Impact of Artificial Intelligence on Employment?" Bruegel (2017).

[8] Sara Gerke, Timo Minssen and Glenn Cohen. "Ethical and legal challenges of artificial intelligence-driven healthcare. Artificial Intelligence in Healthcare, Elsevier Public Health Emergency Collection, (2020).

[9] P.K. Suar, SR Samanta, Going Cashless Behind the Wheels Digital Options for SRTO, Solid State Technology 63 (5) (2020)

[10]Subhajit Basu, Adekemi Omotubora, Matt Beeson, Charles Fox. "Legal framework for small autonomous agricultural robots." $A I$ \& Society, Vol 35, (2020), 113-134.

[11] Thomas Davenport and Ravi Kalakota. "The potential for artificial intelligence in healthcare." Future Healthcare Journal, Vol 6, No 2, (2019), 94-98. 